\title{
SYMPTOM EVOLUTION IN RICE GENOTYPES UNDER IRON TOXICITY CONDITIONS
}

\author{
R. K. Shrestha ${ }^{1}$ and M. Becker ${ }^{2}$ \\ ${ }^{1}$ Institute of Agriculture and Animal Sciences, Tribhuvan University, Nepal \\ ${ }^{2}$ Institute of Crop Science and Resource Conservation, University of Bonn, Germany
}

\begin{abstract}
A greenhouse study was conducted in the University of Bonn, Germany during July-September, 2009 to comparatively evaluate six contrasting lowland rice genotypes, viz. IR31785, Nipponbare, WAS161, CK73, TOX3107 and Pokkali at increasing rates of Fe (II) under both dry and moist atmospheric conditions. Plants were grown in hydroponic culture for 35-days and subjected to 4-day Fe(II) pulses of 5 (control), 500, 1000 and $1500 \mathrm{mg} \mathrm{Fe}^{2+} \mathrm{L}^{-1}$ under conditions of low $(0.3 \mathrm{Kpa})$ and high $(2.4 \mathrm{Kpa})$ vapor pressure deficit. Rice genotypes expressed earlier and intense leaf bronzing symptoms with increasing $\mathrm{Fe}^{2+}$ stress and as the atmosphere became drier. However, genotypes significantly differed with regards to their response to iron stress. Some of the genotypes were able to maintain low leaf bronzing symptoms under higher level of iron stress and high vapor pressure deficit condition.
\end{abstract}

Key Words: Biomass, Hydroponic, Leaf symptom, Oryza sativa, Vapor pressure deficit

\section{INTRODUCTION}

Iron toxicity is a micro-nutritional disorder in lowland-rice production systems (Dobermann and Fairhurst, 2000) and has been reported in South America, Asia, and Africa (Sahrawat, 2004). At global level, it has affected 5 Mha (Million hectare) low land area (Becker and Asch, 2005). Iron toxicity is caused by excessive uptake and acropetal translocation of Fe (II) towards the leaves. The excessive Fe (II) in symplast enhances the production of radical oxygen species (ROS) which causes oxidation of phenols and thereafter membrane damage and the symptoms are manifested as a copper-like coloring called bronzing. Rice yield is reportedly reduced by $12-100 \%$, depending on the severity of toxicity and the tolerance of the rice genotypes (Audebert and Sahrawat, 2000). The severity of iron toxicity depends on type of soil, intensity and duration of Fe(II) stress, genotype and growth stage and uptake and translocation in the plants. The uptake and acropetal translocation of Fe (II) into the leaves is affected by climatic factors such as temperature, air humidity and wind velocity as well as varietal characteristics such as ability to regulate transpiration under stress conditions (Taiz and Zeiger, 2006). With the extended dry season cultivation of lowland rice in intensified production systems and with increasing climate change phenomena affecting the atmospheric conditions (both drought and excess rainfall), higher crop transpiration rates may be expected with possible consequences on Fe (II) translocation patterns in iron toxicity-affected areas. However, there is dearth of information on the effect of seasonal variation on iron toxicity of rice. The aim of the study was a comparative evaluation of the rice cultivars under increasing rates of applied Fe (II) under low and high air moisture conditions.

\section{MATERIALS AND METHODS}

A pot experiment was conducted in greenhouse of the institute of Crop Science and Resource Conservation (INRES), Department of Plant Nutrition, University of Bonn, Germany between July 
to September 2009 using two chambers consisting of contrasting vapor pressure deficit (VPD) conditions. Seeds of six rice genotypes, viz. IR31785, Nipponbare, WAS161, CK73, TOX3107 and Pokkali were pregerminated in petridishes for 48 hours. Four days after seeding (DAS), seedlings were grown in sand for 5 days. The nine-day-old seedlings were transferred into the hydroponic setup filled with $25 \mathrm{~L}$ of $25 \%$ strength of standard Yoshida nutrient solution for five days. Then after, solution strength was increased to $50 \%$ for another 5 days before using full strength solution on $19^{\text {th }}$ days until the plants were subjected to the treatment application. At treatment application, the 36 day-old plants were transferred from the open box system to the individual polythene tubes filled with $280 \mathrm{ml}$ full strength Yoshida solution and adjusted to four levels of Fe (II) stress, namely 0 (control) and 500, 1000, and $1500 \mathrm{mg} \mathrm{Fe}^{2+} \mathrm{L}^{-1}$. Then after, tubes were transferred into the moist $(0.3 \mathrm{Kpa})$ and dry $(2.4 \mathrm{Kpa})$ chambers. Media solutions were changed two days after treatment application. The $\mathrm{pH}$ of the Yoshida solution during whole experiment was adjusted to 5.0 to 5.05. Within the chambers, treatments (six genotypes at four Fe (II) levels) were completely randomized. The experiment was replicated four times over a 2-week period. Symptoms of Fe toxicity on the Y-leaf (fully expanded youngest leaf) were scored by adapting the Standard Evaluation System for scoring for leaf blast (Pyriculariaoryzae) (INGER, 1996). The percentage of leaf area affected by iron-induced leaf spots was translated into a scale of 0 to 10 scale whereby 0 indicates no symptoms and the severity increases with increasing number. A score of 10 indicates that symptoms cover the complete leave blade and leaves are usually dead. The Analysis of Variance of leaf symptom were analyzed by general linear model (GLM) procedure of SPSS software window version 17.0 and treatment means were separated using Tukey test $(\alpha=0.05)$.

\section{RESULTS AND DISCUSSION}

The expression of bronzing symptoms varied as a function of genotype as well as the intensity and duration of the Fe (II) stress; and the effect of VPD was significant. At both VPD, each genotype expressed stronger and earlier leaf symptom at higher level of Fe stresses and the effect of each level of Fe stress among the genotypes was significantly different. Over 4 days stress duration, the mean leaf symptom score was 1.2 and 4.2 at low and high VPD respectively. However, genotypes exhibited distinct trends of symptom expression under different VPD conditions. At low VPD (high air humidity), none of the studied genotypes except WAS161 expressed symptoms at $500 \mathrm{mg} \mathrm{Fe}^{2+}$ $\mathrm{L}^{-1}$ atgiven stress duration. Pokkali expressed symptoms on day 4 at $1500 \mathrm{mg} \mathrm{L}^{-1} \mathrm{Fe}$. Nipponbare showed symptoms on day 4 at $1000 \mathrm{mg} \mathrm{L}^{-1} \mathrm{Fe}$ as well as at $1500 \mathrm{mg} \mathrm{Fe}^{2+} \mathrm{L}^{-1}$. At TOX3107, symptoms started to develop on day 4 at $1000 \mathrm{mg} \mathrm{Fe}^{2+} \mathrm{L}^{-1}$ and on day 3 at $1500 \mathrm{Fe}^{2+} \mathrm{L}^{-1}$. For CK.73, Symptoms development started at day 3 at $1000 \mathrm{mg} \mathrm{Fe}^{2+} \mathrm{L}^{-1}$ already but already at day 1 at $1500 \mathrm{mg} \mathrm{Fe}^{2+} \mathrm{L}^{-1}$. IR317 developed symptoms from day 2 at 1000 and $1500 \mathrm{mg} \mathrm{Fe}^{2+} \mathrm{L}^{-1}$. WAS161 developed symptoms at all levels of Fe Stresses at day 2. In general, the severity of the symptoms was stronger at higher Fe stress levels. 

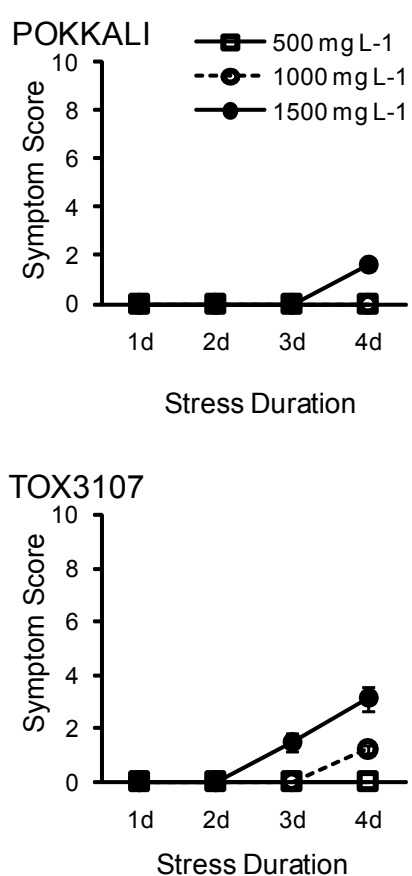
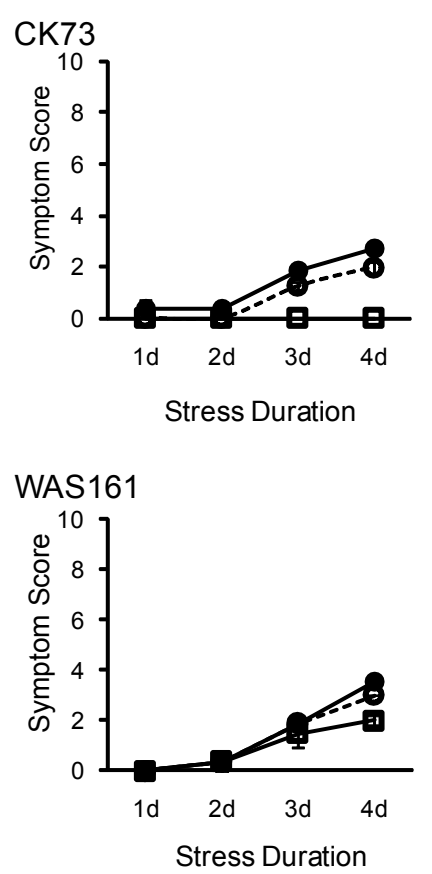
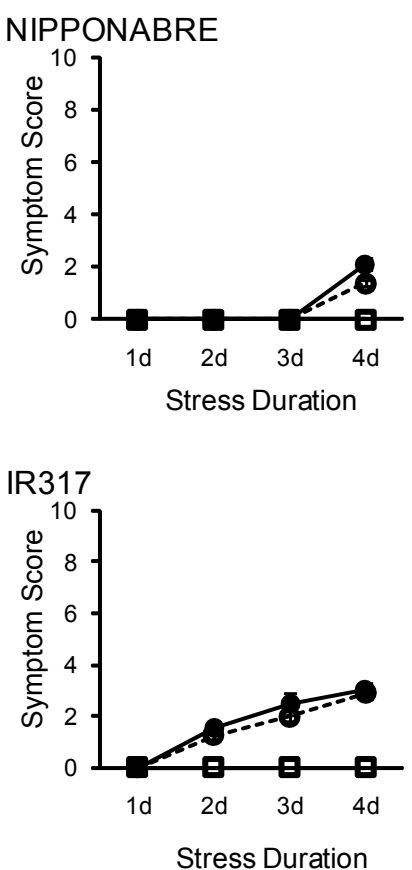

Figure 1: Iron toxicity symptom development of six rice genotypes at low vapor pressure deficit conditions as affected by $\mathrm{Fe}$ (II) added to the solution culture at $500 \mathrm{mg} \mathrm{L}^{-1}, 1000 \mathrm{mg} \mathrm{L}^{-1}$ and $1500 \mathrm{mg} \mathrm{L}^{-1}$ 36-days after seeding and maintained for a duration of four days. Bars represent standard errors of the mean $(n=4)$. When no error bar is visible, the error was smaller than the resolution of the axis.

At $1000 \mathrm{mg} \mathrm{Fe}^{2+} \mathrm{L}^{-1}$, WAS161 and IR317 expressed significantly highest leaf symptoms ( 3 and 2.9 respectively). WAS161 again expressed significantly highest symptoms (3.5) at $1500 \mathrm{mg} \mathrm{Fe}^{2+} \mathrm{L}^{-1}$ (Figure 1).
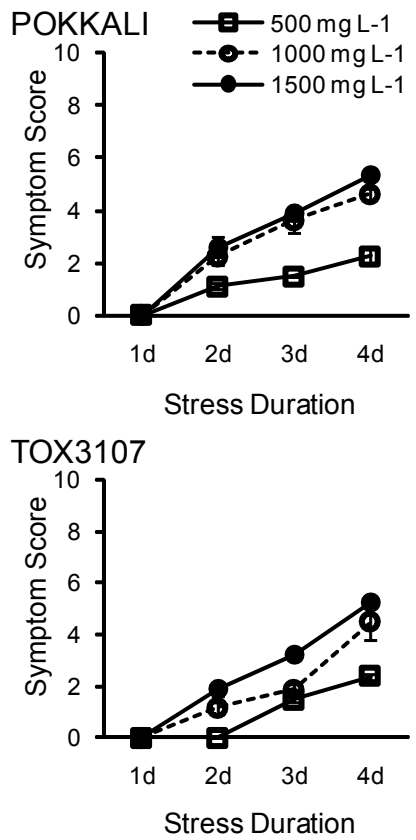
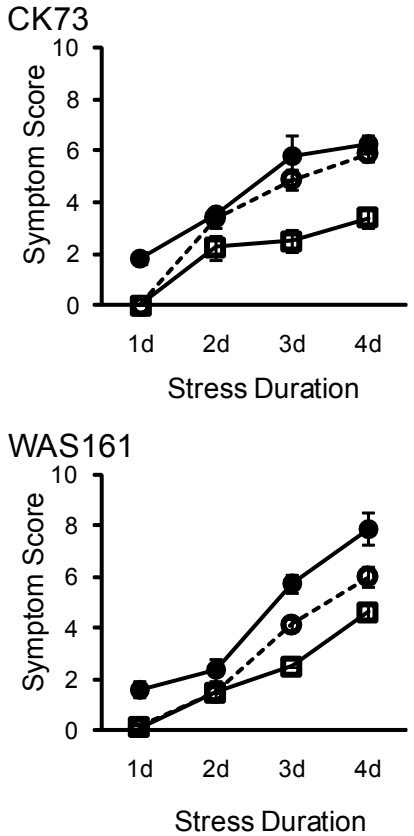
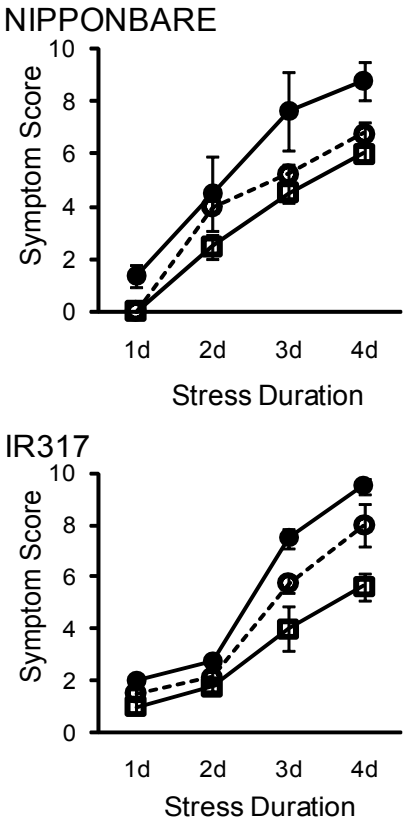

Figure 2: Iron toxicity symptom development of six rice genotypes at high vapor pressure deficit conditions as affected by Fe (II) added to the solution culture at $500 \mathrm{mg} \mathrm{L}^{-1}, 1000 \mathrm{mg} \mathrm{L}^{-1}$ and $1500 \mathrm{mg} \mathrm{L}^{-1} 36$-days after seeding and maintained for a duration of four days. Bars represent standard errors of the mean $(n=4)$. When no error bar is visible, the error was smaller than the resolution of the axis. 
Unlike the low VPD conditions, all genotypes expressed symptoms earlier and the severity of the toxicity symptom expression was higher at the higher intensity of the Fe stress under conditions of high VPD (Figure 2). The severity of the toxicity symptoms was higher in Nipponbare, IR317 and WAS161 as compared to Pokkali, CK73 and TOX3107. Except for CK73, which showed stable toxicity symptom at $1500 \mathrm{mg} \mathrm{Fe} \mathrm{F}^{2+} \mathrm{L}^{-1}$ stress after 3 days, all genotypes expressed high symptoms over the 4-day stress period and their severity is expected to increase further with prolonged Fe stress duration.

This suggests that time of occurrence as well as the severity of the symptoms is significantly affected by intensities of iron in growing medium as well as air humidity conditions. Such type of effect of air humidity condition on leaf symptom expression was also observed by Kponger et al., (2003).

\section{CONCLUSION}

Rice genotypes get more sensitive towards elevated levels of $\mathrm{Fe}$ in the growing media as the atmospheric conditions become drier. The extent of such responses, however, depends on the genotype. Genotypes such as Pokkali and TOX3107 not only tolerate higher levels of reduced iron in the medium but are also less sensitive to an increased in the VPD than other genotypes. Varietal screening activities for iron toxicity tolerance must be not only site-specific but also season specific.

\section{ACKNOWLEDEMENTS}

The data used in this paper were obtained in the course of thesis research of R. Shrestha in the international MS program "Agricultural Sciences and Resource Management in the Tropics and Subtropics-ARTS" of the University of Bonn, Germany. R. Shrestha expresses his gratitude for the stipend from the German Academic Exchange Service (DAAD).

\section{REFERENCES CITED}

Audebert, A., and K. L. Sahrawat. 2000. Mechanisms for iron toxicity tolerance in lowland rice. Journal of Plant Nutrition 23: 1877-1885.

Becker, M. and F. Asch. 2005. Iron toxicity in rice - conditions and management concepts. Journal of Plant Nutrition and Soil Science, 168(4): 558-573.

Dobermann, A., and T. Fairhurst. 2000. Rice: Nutrient disorders and nutrient management. International Rice Research Institute, Manila, The Philippines.

INGER. 1996. Standard evaluation system for rice. (4th Ed.). International Rice Research Institute, Manila, The Philippines.

Kponger, D., P. L. G. Vlek and M. Becker. 2003. Developing a standardized procedure to screen lowland rice (Oryza sativa) seedlings for tolerance to iron toxicity. In: University of Göttingen and ATSAF: Technological and Institutional Innovations for Sustainable Rural Development. Göttingen, Germany: Klartext GmbH. p.103.

Sahrawat, K.L. 2004. Iron toxicity in wetland rice and the role of other nutrients. Journal of Plant Nutrition, 27: 1471-1504.

Taiz, L., and E. Zeiger. 2006. Plant Physiology. (4 $4^{\text {th }}$ Ed.). Sinauer Associates Inc. Publishers, Sunderland, Massachusettes, p65 